\title{
Gustatory Neural Responses in the Medial Orbitofrontal Cortex of the Old World Monkey
}

\author{
Thomas C. Pritchard, ${ }^{1}$ Erin M. Edwards, ${ }^{1}$ Carrie A. Smith, ${ }^{1}$ Kristen G. Hilgert, ${ }^{1}$ Andrew M. Gavlick, ${ }^{1}$ \\ Thomas D. Maryniak, ${ }^{1}$ Gary J. Schwartz, ${ }^{2}$ and Thomas R. Scott ${ }^{3}$ \\ ${ }^{1}$ Department of Neural and Behavioral Sciences, The Pennsylvania State University College of Medicine, Hershey, Pennsylvania 17033 , ${ }^{2}$ Departments of \\ Medicine and Neuroscience, Albert Einstein College of Medicine, Bronx, New York 10461, and ${ }^{3}$ San Diego State University College of Sciences, La Jolla, \\ California 92182
}

The primary taste cortex has widespread and occasionally dense projections to the orbitofrontal cortex (OFC) in the macaque. Nonetheless, electrophysiological studies have revealed that only $2-8 \%$ of the cells in the OFC are activated by taste stimuli on the tongue. We describe an area centered in Brodmann's area 13m of the medial OFC ( $\mathrm{mOFC}$ ) where taste neurons are more concentrated. It consists of a $12 \mathrm{~mm}^{2}$ core, where gustatory neurons constituted $20 \%$ of the population, and a $1 \mathrm{~mm}$ perimeter in which $8 \%$ of the cells responded to taste. Data were collected from three awake cynomolgus monkeys (Macaca fascicularis) prepared for chronic recording. Single neurons were isolated with epoxylite-coated tungsten microelectrodes and tested for responsiveness to $1.0 \mathrm{M}$ glucose, $0.3 \mathrm{M} \mathrm{NaCl}, 0.03 \mathrm{M} \mathrm{HCl}$, and $0.001 \mathrm{M} \mathrm{QHCl}$. These stimuli elicited responses that were $96 \%$ excitatory and ranged from 5.2 to 5.9 spikes/s. Cells were broadly tuned $(\mathrm{H}=$ $0.79)$, similar to those in the anterior insula $(\mathrm{H}=0.70)$, and decidedly unlike the narrowly tuned taste neurons in the caudolateral OFC ( $\mathrm{clOFC} ; \mathrm{H}=0.39$ ). Whereas $82 \%$ of the taste cells in the clOFC respond to glucose, in the $\mathrm{mOFC}, \mathrm{HCl}$-responsive (56\%), glucose-responsive (50\%), NaCl-responsive (43\%), and $\mathrm{QHCl}-$-responsive (40\%) cells were almost evenly represented. The mOFC taste area appears to comprise a major gustatory relay that lies anatomically and functionally between the anterior insula and the clOFC.

Key words: taste; orbitofrontal; cortex; macaque; coding; neurophysiology

\section{Introduction}

In macaque monkeys, cortical processing of gustatory information takes place in the anterior insula (AI), the inner operculum (Scott et al., 1986b; Yaxley et al., 1990; Scott and Plata-Salamán, 1999), and the orbitofrontal cortex (OFC) (Rolls et al., 1989; Rolls and Baylis, 1994). Based on the density of thalamocortical projections, the so-called "gustatory macula" of the primary taste cortex (Bagshaw and Pribram, 1953) is the crescent-shaped area that caps the circular sulcus at the junction of the rostrodorsal insula and the inner operculum (Mesulam and Mufson, 1982; Pritchard et al., 1986; Carmichael and Price, 1995b). Tasteresponsive neurons also are located within the body of the insula (Yaxley et al., 1990; Scott et al., 1991), which receives most of its afferent projections from the insular-opercular crescent rather

Received Feb. 1, 2005; revised May 10, 2005; accepted May 13, 2005.

This work was supported by United States Public Health Service Grant DK59549. Preliminary results were presented at the 2002 Australasian Association for ChemoSensory Sciences, Heron Island, Australia; at the 2002 Annual Meeting of the Society for Neuroscience, San Diego, CA; and at the 2004 International Symposium on Olfaction and Taste, Kyoto, Japan. Localization of the recording chamber was assisted by magnetic resonance images graciously provided by Drs. Michael Smith and Qing Yang. Kathy Smith assisted with the histological preparation of the brain Expert technical assistance was provided by Andrew Petticoffer and Erin Nedderman. Amanda III and Rebekah Greenebaum trained the monkeys and helped with the data analysis. They have our gratitude.

Correspondence should be addressed to Dr. T. C. Pritchard, Department of Neural and Behavioral Sciences, The Pennsylvania State University College of Medicine, H181, 500 University Drive, Hershey, PA 17033. E-mail: tcp1@psu.edu.

D01:10.1523/JNEUROSCI.0430-05.2005

Copyright $\odot 2005$ Society for Neuroscience $\quad$ 0270-6474/05/256047-10\$15.00/0 than directly from the thalamus (Mesulam and Mufson, 1982; Mufson and Mesulam, 1982).

The available anatomical evidence suggests that gustatory information ascends from both the insular-opercular crescent and the body of the insula to the OFC along a multisynaptic pathway that courses through the ventral dysgranular and agranular insula (Carmichael and Price, 1994, 1995a,b; Baylis et al., 1995; Pritchard and Norgren, 2004). Taste cells have been found within the OFC as far medially as Brodmann's area 14 (BA 14), which lies adjacent to the midline, and as far laterally as BA $47 / 120$, which forms the boundary between the OFC and the lateral convexity (Rolls et al., 1989; Rolls and Baylis, 1994). These electrophysiological studies have focused on a variety of issues, but all report that taste neurons represent only a small fraction $(2-8 \%)$ of the cells within the OFC (Rolls et al., 1990, 1999).

Anatomical studies by Carmichael and Price (1994) suggest that taste neurons should be prevalent within BAs 131 and $13 \mathrm{~m}$. BA 131 receives afferent projections from the primary taste cortex as well as direct and indirect projections from the ventral agranular insula via BA 13m (Carmichael and Price, 1995b, 1996). Thus, the anatomical literature provides a firm basis for the widespread distribution of taste-responsive neurons within the OFC that has been reported by Rolls and Baylis (1994). By the same token, these same studies suggest that taste neurons should constitute $>2-8 \%$ of the cells within the posterior OFC, and especially within BAs $13 \mathrm{~m}$ and 131 .

The present study describes a circumscribed region within 
and adjacent to BA $13 \mathrm{~m}$ of the OFC in the macaque where taste neurons represent an unprecedented $7-28 \%$ of the population. This high concentration of taste cells, combined with their unique response properties, support our assertion that the medial OFC (mOFC) contains a significant cortical taste projection area, the scope and qualities of which have not been described previously.

\section{Materials and Methods}

Subjects. The subjects were three male cynomolgus monkeys (Macaca fascicularis) weighing $5.0-6.4 \mathrm{~kg}$ at the beginning of the experiment. Each monkey was housed individually in a primate cage of suitable size in a climate-controlled room (lights on 7:00 A.M. to 7:00 P.M.). The monkeys had ad libitum access to water and Harlan Teklad (Madison, WI) monkey chow 2050, except for the $17 \mathrm{~h}$ immediately preceding the recording session. This protocol was approved by the Institutional Animal Care and Use Committee of The Pennsylvania State University College of Medicine. The research was in accordance with the guidelines of the Society for Neuroscience as described in Policies on the Use of Animals and Humans in Neuroscience Research (1995).

Training and preoperative treatment. Before surgery, each monkey was adapted to a primate chair specifically designed for neurophysiological recording. During training, the monkey was reinforced with fruit and fruit juice for sitting quietly in the chair. Surgery was scheduled after the monkey had learned to enter the chair eagerly and sit quietly. After surgery and a $14 \mathrm{~d}$ recovery period, the monkey was returned to the chair and acclimated to the head restraint apparatus. Neurophysiological recording began after the monkey became accustomed to sitting with its head restrained; it was otherwise free to move and typically adopted a relaxed position. The monkey was reinforced periodically with fruit juice during the recording sessions, which were limited to $6 \mathrm{~h}$ but terminated sooner if the monkey showed signs of discomfort.

Anesthesia and surgery. Surgery to attach the chronic recording chamber to the skull was performed under sterile conditions in the primate surgical suite. Placement of the recording chamber was guided by cranial $\mathrm{x}$-rays (anteroposterior and mediolateral perspectives) taken before surgery. After injections of atropine sulfate $(0.04 \mathrm{mg} / \mathrm{kg}, \mathrm{i} . \mathrm{m}$.) and ketamine hydrochloride (10 mg/kg, i.m.), the monkey was weighed and shaved. A local anesthetic (Cetacaine) was applied topically to the larynx before insertion of an endotrachial tube. Anesthesia was induced with $1.5 \%$ halothane in a nitrous oxide/oxygen mixture (67\%:33\%, $3 \mathrm{~L} / \mathrm{min}$ ) and maintained at $1.0-1.5 \%$ for the duration of the procedure. The core temperature was held between 35 and $36^{\circ} \mathrm{C}$, and an electrocardiogram was monitored on an oscilloscope and audio speaker. End-tidal $\mathrm{CO}_{2}$ was maintained within the physiological range of 35-50 torr. Dextrose (5\%) and lactated Ringer's solution was delivered intravenously at $4 \mathrm{ml} / \mathrm{kg} / \mathrm{h}$.

Chamber placement was guided by presurgical radiographs (anteroposterior and mediolateral planes) and a magnetic resonance imaging (MRI) series. These films allowed us to locate the anterior clinoid process of the sphenoid bone relative to ear bar zero, which was the reference we used during surgery. With the monkey positioned in a Kopf stereotaxic frame, the scalp was incised and a stainless steel recording chamber (30 $\mathrm{mm}$ outer diameter) was secured to the skull with orthopedic screws and dental acrylic. A stainless steel ground wire was wrapped around the skull screws and externalized to a gold Amphenol pin mounted in a plastic receptacle. Three stainless steel wires $(0.010$ inch) lowered and fixed 20 $\mathrm{mm}$ into the brain were used as fiduciary marks on the $\mathrm{x}$-rays that were taken at the bottom of each recording track. Two stainless steel tubes ( 7 $\mathrm{mm}$ inner diameter, $12 \mathrm{~mm}$ outer diameter, $6.7 \mathrm{~cm}$ long) were mounted transversely on the monkey's skull behind the recording chamber. During recording, the monkey's head was secured to the primate chair with stainless steel rods that passed through each tube and clamped to the chair. At the completion of the surgery, nitrofurazone was applied topically to the wound margin. To prevent infections, enrofloxacin (Baytril; $45 \mathrm{mg}$; Bayer Corporation, Shawnee Mission, KS) was administered orally for $10 \mathrm{~d}$. Buprenorphine $\mathrm{HCl}(0.01 \mathrm{mg} / \mathrm{kg}$, i.m.; Reckitt Benckiser Healthcare, Richmond, VA) was given as an analgesic at the beginning of surgery and every $12 \mathrm{~h}$ thereafter for $2 \mathrm{~d}$. Before collection of electrophys- iological data, the monkey was reanesthetized, and the skull overlying the target recording area was removed.

Electrophysiological recording. The search for the gustatory responsive area within the OFC was guided by cranial x-rays and by MRI films ( 3 $\mathrm{mm}$ resolution) obtained before surgery. Epoxylite-coated microelectrodes (Frederic Haer, Bowdoinham, $\mathrm{ME})(\mathrm{Z}=2-4 \mathrm{M} \Omega$ at $1 \mathrm{kHz})$ were used to isolate the activity of individual neurons. The microelectrodes were heat sterilized and placed in a sterile 21 ga stainless steel guide tube that both penetrated the dura and provided lateral support for the delicate electrodes during their descent to the OFC. The electrode/guide tube assembly was secured to a Narishige (Tokyo, Japan) $x-y$ stage/hydraulic microdrive (MO-951) that mounted to the top of the recording chamber. After the dura was anesthetized ( $0.3 \mathrm{ml}$ of Lidocaine), the guide tube was inserted $\sim 5 \mathrm{~mm}$ into the brain. The microelectrode was advanced with the gross manual drive to within $5 \mathrm{~mm}$ of the OFC; the hydraulic microdrive was used to advance the microelectrode for the remainder of the track. Mapping of the OFC was done using a $1 \mathrm{~mm}$ grid search pattern.

We used conventional electrophysiological recording techniques for differential amplification and display of the neural signal. The amplitude of taste neurons ranged from 200 to $2000 \mu \mathrm{V}$, which provided a minimum signal/noise ratio of 4:1. A window discriminator coupled to an analog delay device $(2 \mathrm{~ms}$ ) enabled the waveform of each action potential to be monitored during data collection. Action potentials with a consistent time course and amplitude were judged to be from a single neuron. The neural data and voice commentary were digitized and stored on computer (Spike2; Cambridge Electronic Design, Cambridge, UK) and copied to a compact disc for later analysis. At the bottom of each electrode track, $\mathrm{x}$-rays were taken in the anteroposterior and mediolateral planes. The x-ray films of the microelectrode, the three implanted fiduciary wires, and the bony landmarks of the skull base were used to confirm and correct, if necessary, the placement of the electrode, and to guide mapping of the OFC over the term of the project. After each recording session, the recording chamber was flushed with sterile saline, treated with nitrofurazone, and closed with a sterile cap.

Sensory stimulation. The data of this study were derived from application of the four prototypical stimuli: $0.3 \mathrm{M}$ sodium chloride $(\mathrm{N}$ or $\mathrm{NaCl})$, $1.0 \mathrm{~m}$ glucose $(\mathrm{G}), 0.03 \mathrm{~m}$ hydrochloric acid $(\mathrm{H}$ or $\mathrm{HCl})$, and $0.001 \mathrm{M}$ quinine hydrochloride ( $\mathrm{Q}$ or $\mathrm{QHCl})$. If the recording remained stable, an extended array of stimuli was applied, the responses to which will be presented in a separate report. Each sapid stimulus was administered at room temperature as a $0.5 \mathrm{cc}$ bolus from a plastic $1 \mathrm{cc}$ syringe with its tip cut off. Each stimulus was preceded by at least one application of distilled water $\left(\mathrm{dH}_{2} \mathrm{O}\right)$ and followed by at least one $\mathrm{dH}_{2} \mathrm{O}$ rinse $(1 \mathrm{ml})$ and an interstimulus interval of at least $45 \mathrm{~s}$. A longer rest period and additional rinses were included if spontaneous activity had not returned to baseline.

Each neuron also was tested with warm $\left(37^{\circ} \mathrm{C}\right)$ and cold $\left(17^{\circ} \mathrm{C}\right) \mathrm{dH}_{2} \mathrm{O}$. Sensitivity to olfactory stimulation was tested with five odorants: geraniol, benzaldehyde, iso-amylacetate, limonene, and phenethyl alcohol. A cotton-soaked pledget of each odorant was placed in a $250 \mathrm{ml}$ squeeze bottle. The spout of the bottle was placed $20 \mathrm{~cm}$ in front of the monkey's face, and the odorant was expelled with a single squeeze. The response elicited by each odorant was compared with that evoked by a comparable application of clean air. Tactile stimulation of the tongue, face, lips, gingivae, and teeth was done routinely, but probing of the tongue typically could not be done with precision. There was no specific protocol for testing visual responsiveness, but we took note of neurons that changed their firing rate when the experimenter turned and faced the monkey, presented the fluid syringe, etc. Neurons that responded reliably to movements such as these were designated as being responsive to visual stimulation.

Histological procedures and location of recording sites. Two of the three monkeys were killed, and their brains were processed histologically to determine the precise locations of data collection. After the final recording session, an injection of 100-200 $\mathrm{nl}$ of wheat germ agglutinin-horseradish peroxidase (HRP) (L3892; Sigma, St. Louis, MO) was made under $\mathrm{x}$-ray guidance at the center of the taste-responsive area. Three days later, a grid of marker lesions ( $20 \mu \mathrm{A}$ for $20 \mathrm{~s}$, electrode negative) was made in the area surrounding the taste-responsive core. X-rays taken during lesioning were used so that the recording sites could be reconciled with the 
histological material. After placement of the marker lesions, each monkey was sedated with ketamine hydrochloride $(10 \mathrm{mg} / \mathrm{kg}$, i.m. $)$, administered a lethal dose of sodium pentobarbital $(75 \mathrm{mg} / \mathrm{kg}$, i.p.), and perfused transcardially with normal saline, followed by $3 \%$ paraformaldehyde and two sucrose solutions $(10 \%, 20 \%)$, each dissolved in phosphate buffer. The brains were blocked in the stereotaxic plane (in situ), removed from the skull, and submerged in successive $20 \%$ and $30 \%$ sucrose solutions. Frozen $50 \mu \mathrm{m}$ coronal sections were arranged into four series and mounted on gelatin-coated slides. Two series were reacted for HRP (Gibson et al., 1984); one was stained with neutral red, and the other was coverslipped without staining. The two unreacted series were stained with cresyl violet or processed for parvalbumin (Carmichael and Price, 1994). Although the HRP reaction product was clearly visible at the injection site, there was little anterograde or retrograde transport. Failure to detect HRP transport was probably caused by our use of paraformaldehyde without glutaraldehyde for fixation (J. L. Price, personal communication).

The neutral red, cresyl violet, and parvalbumin material was used to determine BA boundaries within the posterior OFC. Using the cytoarchitectonic criteria of Carmichael and Price (1994), each BA was plotted on photographs of coronal sections of each monkey's brain. One BA map of the OFC was constructed for each monkey by measuring the distance of each cytoarchitectural boundary (through layer IV of the OFC) from a point $5 \mathrm{~mm}$ lateral to the midline. Charting the BA boundaries from a fixed point close to our recording area minimized the local distortion that takes place when a gyrencephalic area of the brain is reconstructed as a two-dimensional map.

Data analyses. The spontaneous activity of each neuron was measured during the latter part of the interstimulus interval. Evoked responses were counted during the $5 \mathrm{~s}$ period after stimulus onset. Responses to sapid stimuli that exceeded the mean response to $\mathrm{dH}_{2} \mathrm{O}$ by $1.96 \mathrm{SD}(95 \%$ confidence interval) were significant. By comparing the taste-evoked response to the water-evoked response instead of the spontaneous level of activity, we were able to control for possible visual, somatomotor, thermal, and anticipatory activation associated with the presentation and consumption of the fluid stimuli. This is an important consideration when using an awake, behaving preparation. Responses to $\mathrm{dH}_{2} \mathrm{O}$ that exceeded the mean spontaneous rate by $1.96 \mathrm{SD}$ were significant.

Primary analyses included (1) the mean response to each stimulus, (2) the number of stimuli to which each neuron responded with excitation or inhibition, (3) the proportion of neurons responding to each stimulus, (4) breadth of tuning (BOT) (Smith and Travers, 1979), and (5) quantitative measures of reliability to the repeated application of the same stimulus.

Derived analyses included (1) interstimulus and interneuronal correlation coefficients, (2) a neuron cluster analysis, and (3) a multidimensional neuron space. A detailed description of multidimensional scaling and cluster analysis can be found in the study by Bieber and Smith (1986).

\section{Results}

\section{Location and extent of the gustatory region}

The cytoarchitectural description of the taste-responsive region in the mOFC is based on the results obtained from two of the three monkeys in the project. One monkey (SAT2) provided 65\% of the electrophysiological data, so the more detailed map of the mOFC taste area was obtained from his brain.

Gustatory neurons were collected from a $20 \mathrm{~mm}^{2}$ area, the center of which was located $\sim 8 \mathrm{~mm}$ lateral to the midline and $\sim 6$ $\mathrm{mm}$ rostral to the anterior clinoid process of the sphenoid bone (Fig. 1). Taste neurons constituted $19.7 \%$ of the cells in the 12 $\mathrm{mm}^{2}$ center of this region and $7.8 \%$ of the neurons in the adjacent $1 \mathrm{~mm}$ perimeter. The core area straddled the lateral half of BA 13m and the rostral half of BA 13a but encroached on adjacent parts of BA $13 \mathrm{~b}$ and the medial agranular insula (Fig. 2). The perimeter taste area included the medial edges of BAs 131 and $12 \mathrm{~m}$, the caudal portion of BA $11 \mathrm{~m}$, and the rostromedial por-
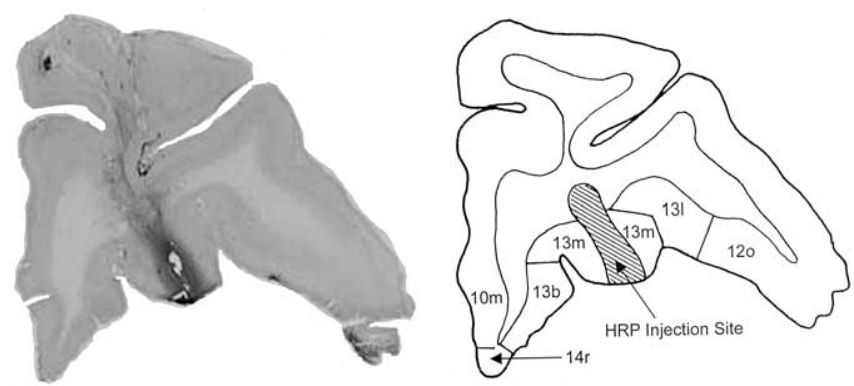

Figure 1. Photomicrograph (left) and diagram (right) of a coronal section of the mOFC in monkey SAT2. The HRP injection in the right panel marks the location of the core taste area in BA $13 \mathrm{~m}$.

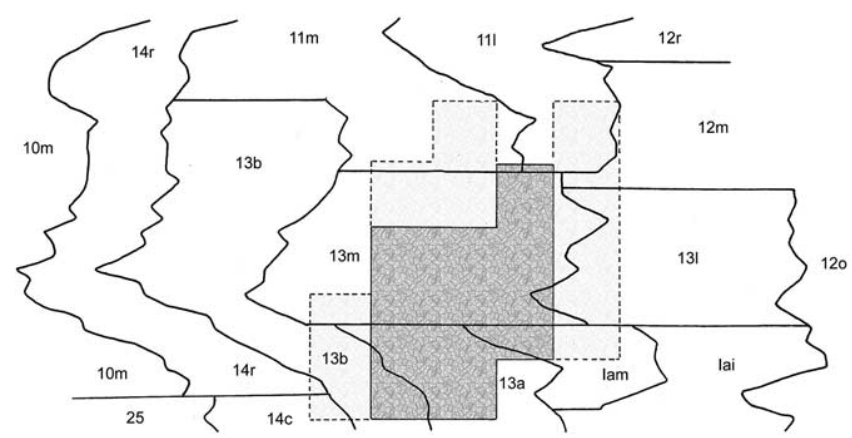

Figure 2. The core and perimeter taste areas projected onto an unfolded map of the OFC of monkey SAT2. Distances between BA boundaries were measured through layer IV.

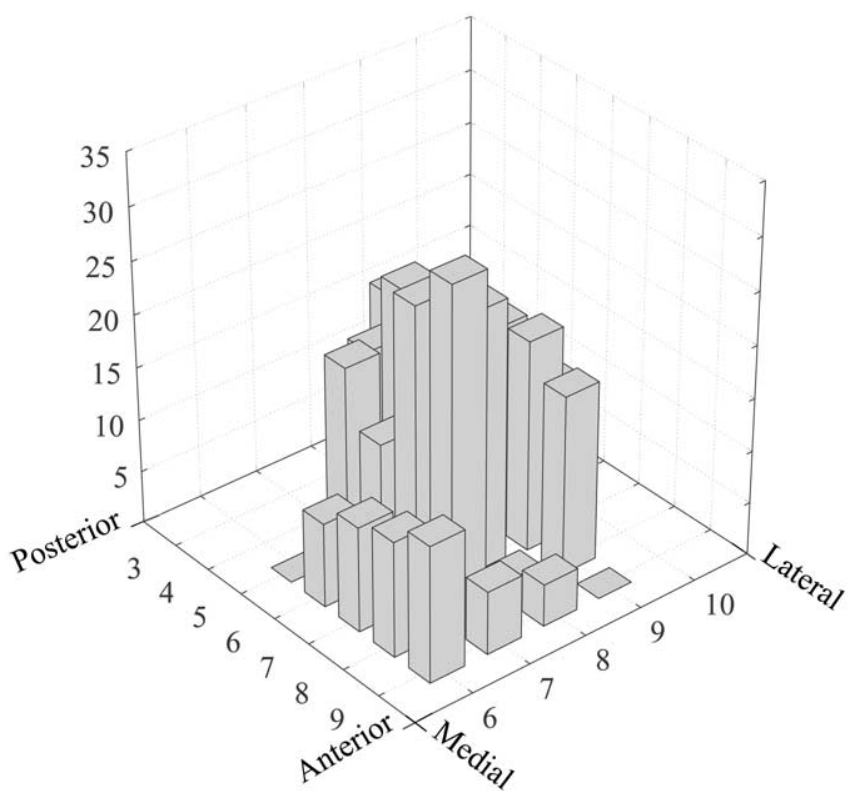

Figure 3. Three-dimensional histogram showing the percentage of taste cells recorded within the $\mathrm{mOFC}$ search grid of monkey SAT2. The anterior/posterior and medial/lateral coordinates (in millimeters) are indexed to the anterior clinoid process of the sphenoid bone.

tions of BAs $13 \mathrm{~m}$ and $13 \mathrm{~b}$. The width of the perimeter taste area was at least $1 \mathrm{~mm}$, beyond which the proportion of taste cells did not warrant further exploration (Fig. 3).

\section{Sensory demographics within the mOFC}

During 321 recording tracks made in three monkeys, 242 (12.5\%) of the 1943 neurons tested responded significantly to one or more 
of the gustatory stimuli. As described above, these taste neurons were not evenly distributed across the posterior OFC. Time permitting, they were tested with olfactory stimuli ( $n=967$ cells) and with perioral tactile ( $n=784$ cells) and thermal $(n=746$ cells) stimulation. Cells that responded to visual stimuli such the experimenter's movements also were noted. Figure 4 shows the record of two neurons that responded to $\mathrm{HCl}$, benzaldehyde, and $\mathrm{dH}_{2} \mathrm{O}$.

Of the 242 taste cells, $196(81 \%)$ responded exclusively to gustatory stimuli. The remaining 46 gustatory neurons showed side-band sensitivity to one or more of the other sensory stimuli, with bimodal sensitivity being the most common (38 of 46 cells). Twenty-three $(61 \%)$ of the 38 bimodal taste neurons responded to olfactory stimulation.

When a cell in the mOFC responded to only one modality, taste was the dominant sensory presence (Table 1). Whereas $10.1 \%$ of the tested cells were exclusively gustatory, just $2.6 \%$ responded only to tactile, $0.9 \%$ to thermal, $0.9 \%$ to visual, and $0.7 \%$ to olfactory stimulation. Another $0.7 \%$ responded exclusively to the application of $\mathrm{dH}_{2} \mathrm{O}$. An additional $1.7 \%$ discharged reliably as the syringe approached the monkey's mouth. These anticipatory neurons fired regardless of whether taste or $\mathrm{dH}_{2} \mathrm{O}$ was being delivered. The adequate stimulus for these anticipatory responses could not be determined.

\section{Characteristics of taste cells \\ Distribution}

Multineuron taste responses were observed at 11 of the 12 coordinates within the core taste area and 3 of the 20 coordinates within the perimeter. This reinforces our observation that taste cells composed a higher proportion of the neurons in the mOFC core than in the primary taste cortex (Scott and Plata-Salamán 1999) or in the caudolateral OFC (clOFC) (Rolls et al., 1990), where multiunit responses have not been reported.

\section{Spontaneous and water-evoked activity}

Of the total of 242 taste cells, 100 were excluded from the coding analyses because of incomplete data $(n=43)$ or because they gave responses only to tastants that were beyond the four basic stimuli being considered here $(n=57)$. The remaining 142 gustatory neurons, the activity of which serves as the basis for the body of this report, had a mean spontaneous discharge rate of $3.1 \pm 5.6$ spikes/s (range, $0.0-46.4$ spikes/s). The average response to $\mathrm{dH}_{2} \mathrm{O}$ was $3.9 \pm 6.9$ spikes/s (range, $0.0-61.0$ spikes/s).

\section{Reliability of responses}

Application of the basic taste stimuli and testing for sensitivity to other modalities typically required $\sim 20 \mathrm{~min}$. For 80 neurons, the isolation remained unequivocal, so 124 of the 258 (48\%) significant taste responses were retested. The test-retest reliability of these cells was determined by calculating the Pearson productmoment correlation coefficient between responses to the two sets of applications. If the responses from each of the 124 pairs of applications had been identical, the correlation coefficient would have been +1.00 ; we obtained a correlation coefficient of +0.96 , indicating a high degree of stability in the recordings, reproducibility of stimulus applications, and consistency in the monkeys' taste responses.

\section{Excitation and inhibition}

Gustatory responses in the OFC were primarily excitatory. Of the 568 stimulus-neuron interactions (142 neurons by 4 stimuli), $258(45 \%)$ resulted in responses that satisfied the criterion for excitation; 10 (2\%) responded with inhibition, and 300 (53\%)
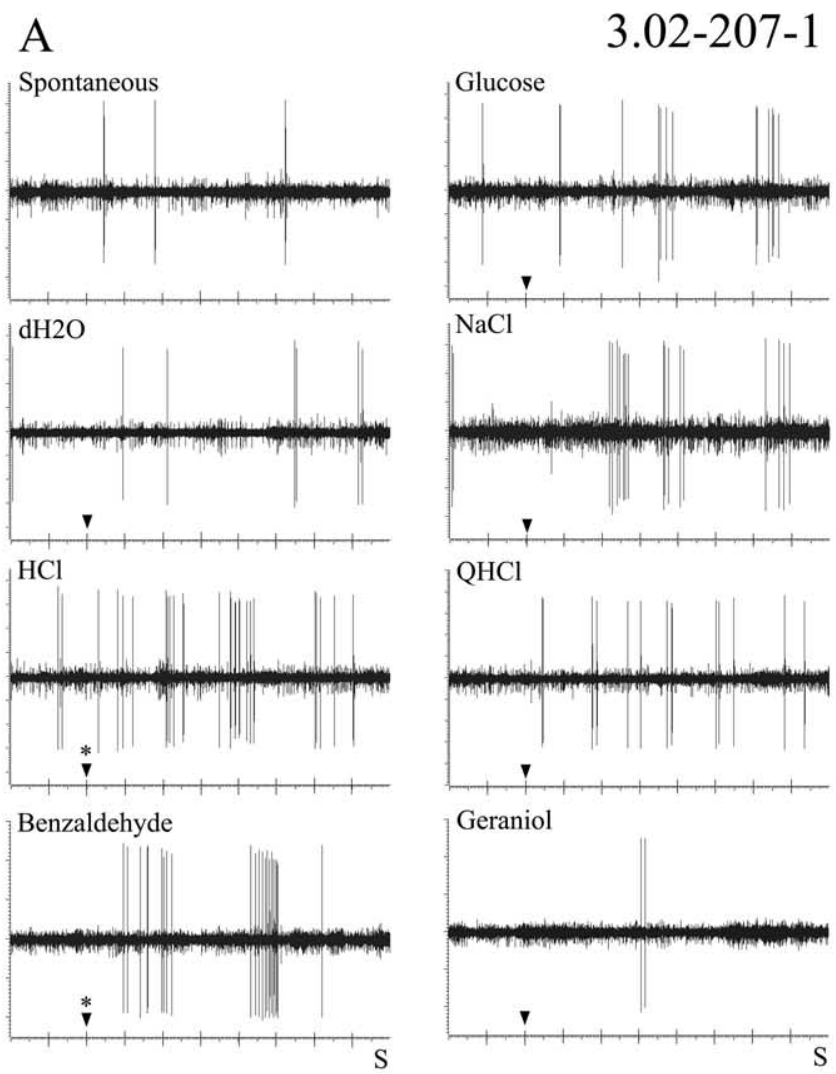

B
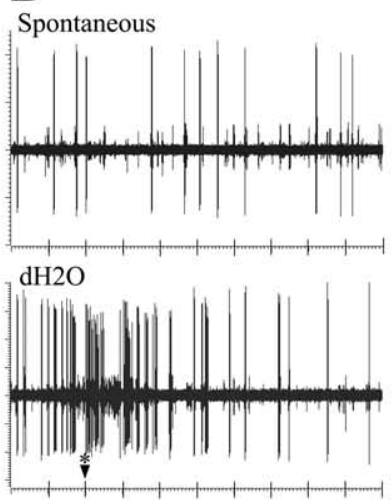

$\mathrm{HCl}$

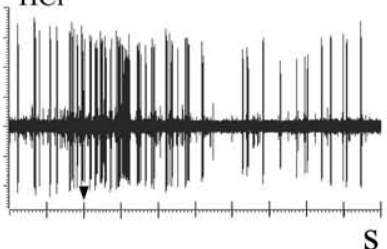

2.02-095-1

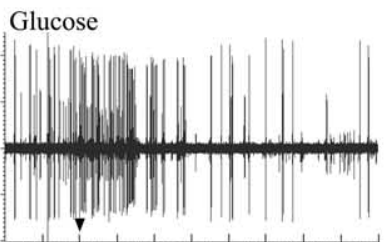

$\mathrm{NaCl}$

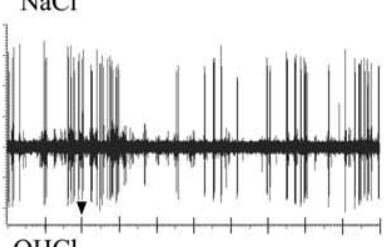

$\mathrm{QHCl}$

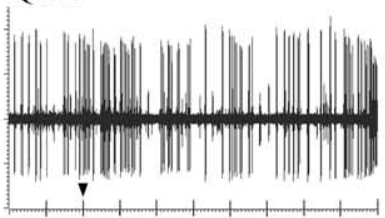

$\mathrm{S}$

Figure 4. Evoked responses from two neurons recorded in the mOFC. $A$, The neuron responded to $\mathrm{HCl}$ and benzaldehyde. $\boldsymbol{B}, \mathrm{A}$ neuron that responded during the application of distilled water $\left(\mathrm{dH}_{2} \mathrm{O}\right)$. The responses to the sapid stimuli were not significantly different from the water-evoked response. Fluid onset is marked by the arrowhead. Significant responses are indicated by asterisks.

gave no response. Although the number of inhibitory responses was within the range of error permitted by the criterion, two cells responded so consistently with inhibition that this appeared to be a true representation of their response to the stimulus array. 
Table 1. Percentage of neurons exhibiting sensory convergence

\begin{tabular}{|c|c|c|c|c|c|}
\hline & Gustatory & Olfactory & Tactile & Thermal & Visual \\
\hline Gustatory & 196/1943 (10.1\%) & 23/967 (2.4\%) & $7 / 784(0.9 \%)$ & $6 / 746(0.8 \%)$ & $2 / 1943(0.1 \%)$ \\
\hline Olfactory & $23 / 967(2.4 \%)$ & $7 / 967(0.7 \%)$ & $2 / 736(0.3 \%)$ & $0 / 725(0.0 \%)$ & 1/967 (0.1\%) \\
\hline Tactile & $7 / 784(0.9 \%)$ & $2 / 736(0.3 \%)$ & $20 / 784(2.6 \%)$ & $2 / 725(0.3 \%)$ & 1/784 (0.1\%) \\
\hline Thermal & $6 / 746(0.8 \%)$ & $0 / 725(0.0 \%)$ & $2 / 725(0.3 \%)$ & $7 / 746(0.9 \%)$ & $0 / 746(0.0 \%)$ \\
\hline Visual & $2 / 1943(0.1 \%)$ & $1 / 967(0.1 \%)$ & $1 / 784(0.1 \%)$ & $0 / 746(0.0 \%)$ & $17 / 1943(0.9 \%)$ \\
\hline Multimodal $^{a}$ & $8 / 711(1.1 \%)$ & $8 / 711(1.1 \%)$ & $7 / 711(1.0 \%)$ & 4/711 (0.6\%) & $2 / 711(0.3 \%)$ \\
\hline Total number of neurons & 242 & 41 & 39 & 19 & 23 \\
\hline
\end{tabular}

Each fraction is the number of responsive neurons divided by the number of neurons tested with the respective stimuli.

${ }^{a}$ Nine neurons responded to more than two sensory modalities. Because these neurons had multiple sensitivities, they are included in multiple columns.

\section{Breadth of tuning}

A fundamental characteristic of taste cells is their breadth of sensitivity across a range of taste qualities. One measure of breadth is the number of basic taste stimuli to which each neuron responds. Among the 142 neurons in this study, 62 (44\%) responded to only one of the four basic stimuli, 43 (30\%) responded to two stimuli, $28(20 \%)$ responded to three stimuli, and $9(6 \%)$ responded to all four stimuli. Response breadth is influenced, in large part, by the response criterion used. To avoid such idiosyncrasies and to standardize the measure, the entropy coefficient (H) was calculated for each cell (Smith and Travers, 1979). The proportion of the total response of a neuron that is accorded to each of the basic stimuli is converted to a coefficient that ranges from 0.00 , when all of the action potentials are evoked by a single stimulus, to 1.00 , when the evoked activity is distributed equally across all four stimuli. Because our analysis was based on "net spike activity," we had to make some accommodation for negative numbers, which cannot be used in the entropy formula. The entropy coefficients were calculated using the absolute value of the net response of each cell, based on our assumption that both negative and positive numbers have the same absolute impact on the nervous system (Miyaoka and Pritchard, 1996). The mean BOT for the 142 taste cells in this study was $0.79 \pm 0.15$ (range, $0.28-0.98$ ). The breadth of the taste neurons within the mOFC is reflected in the percentage of cells that responded best to glucose (27\%), $\mathrm{NaCl}$ (25\%), $\mathrm{HCl}(30 \%)$, and $\mathrm{QHCl}(14 \%)$.

The mean response to the signature stimulus in each of the best-stimulus groups fell in a narrow range (5.2-5.9 spikes/s), and for each group, the defining stimulus contributed a narrow majority of the total spikes evoked by all four of the basic taste stimuli (range, 51-63\%) (Fig. 5).

Topographic distribution of taste sensitivity

One basic strategy for discriminating among taste qualities might be to represent them in physically separate areas of the cortex (i.e., to organize quality topographically). We examined this issue in two ways. First, the $12 \mathrm{~mm}^{2}$ core region of one monkey was divided into anteromedial, anterolateral, posteromedial, and posterolateral quadrants. A $\chi^{2}$ analysis on the proportion of G-best cells, N-best cells, etc., among quadrants revealed no significant differences. Then, we calculated the average coordinate within the mOFC recording grid for each class of best-stimulus cell and, once again, found no evidence to suggest a chemotopic organization. These data demonstrate that the mOFC, in accordance with every other level of the primate gustatory system that has been studied electrophysiologically, does not use chemotopic organization to code taste quality (Scott et al., 1986a,b; Pritchard et al., 1989).

\section{Responses to basic stimuli}

The mean response to the basic stimuli across all taste cells was 5.9 spikes/s (uncorrected for $\mathrm{dH}_{2} \mathrm{O}$ ), which was only 2.0 spikes/s
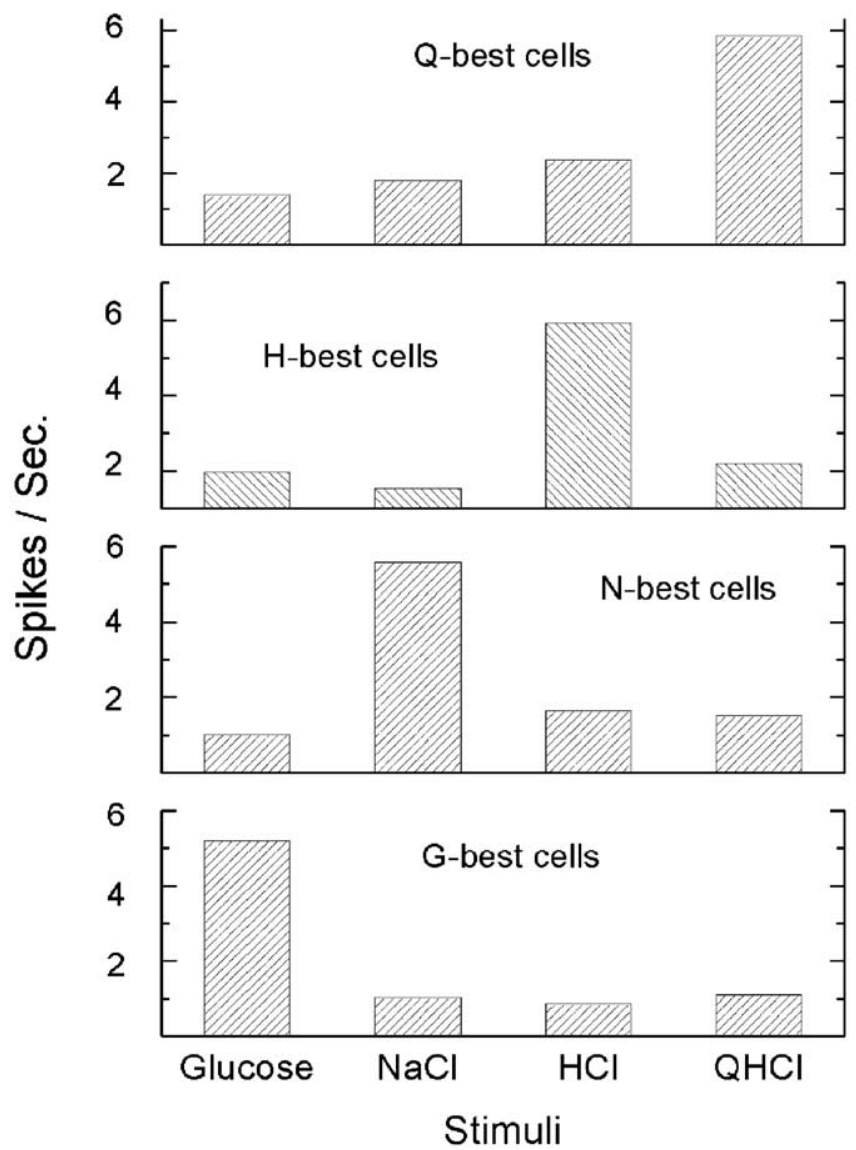

Figure 5. Average response rate for G-, N-, H-, and Q-best cells.

above the evoked rate for water. The mean responses (corrected for $\mathrm{dH}_{2} \mathrm{O}$ ) for each of the basic stimuli were both low and limited in their range (in spikes per second): $\mathrm{G}=2.0 ; \mathrm{N}=1.8 ; \mathrm{H}=2.4$; $\mathrm{Q}=1.7$. Of 258 excitatory responses across all neurons and stimuli (see above), only 24 exceeded 10 spikes/s, which indicates that $\mathrm{mOFC}$ taste neurons operate within a narrow range of discharge frequencies. These 24 neurons were distributed almost equally across the four best-stimulus classes (G-best $=5$; N-best $=6$; H-best $=8$; Q-best $=5$ ).

\section{Neuron types}

There are several morphological, developmental, and functional criteria by which neurons may be classified (Boudreau and Alev, 1973; Whitehead and Frank, 1983; Davis and Jang, 1988; Travers, 1988). The data presented here provide functional characteristics, including discharge rates, $\mathrm{BOT}$, and response profiles. If the response profiles of these gustatory cells across the basic stimuli 
fell into a small number of discrete groups, it would represent cogent evidence for the existence of functionally distinct neuron types in the mOFC.

Using the response profiles of these cells to the four basic stimuli, we calculated the Pearson product-moment correlation coefficients between all pairs of profiles for 142 cells $[n=(142 \times$ 141) $/ 2=10,011$ coefficients] [Because correlation coefficients were used to gauge response similarity, two neurons with responses that were exclusively inhibitory were misplaced in the dendrogram. In both cases, neuron placement was influenced primarily by the greatest response (i.e., most positive) of the cell. For this reason, cells 106 and 084, which were GH-best and NGbest cells, were placed in the Q- and H-clusters, respectively.] This correlation matrix, which provided a measure of relative functional similarity among neurons, was subjected to a cluster analysis. Neurons, numbered in the order in which they were isolated, were grouped into clusters using the average amalgamation method; the results are summarized as a dendrogram (Statistica, version 5.5) (Fig. 6). The most similar pairs of neurons are interconnected first, with additional neurons and groups of neurons being added as an iterative process that was complete when all 142 neurons were connected.

The 142 neuronal profiles represented in Figure 6 are divisible into four large, statistically different groups, corresponding to the four basic taste stimuli. Beginning at the top of the dendrogram, these are as follows: Q-group ( $n=26$; cells 001-114); H-group ( $n=29$; cells 005-089); N-group ( $n=33$; cells 002-031); G-group ( $n=54$; cells 003-135). There were statistically significant subgroups within the $\mathrm{H}-, \mathrm{N}-$, and glucose groups as well. In the H-group, the top 8 neurons (cells 005-109) showed sideband sensitivity to $\mathrm{NaCl}\left(t_{(26)}=3.72 ; p<0.001\right)$ that the remaining 20 neurons (cells 013-089) lacked. The entropy coefficients of these two $\mathrm{H}$-subclusters were 0.86 and 0.69 , respectively $\left(t_{(26)}=3.10 ; p<0.005\right)$. The $\mathrm{N}$-group contained two subclusters, only one of which had significant side-band sensitivity to $\mathrm{HCl}$ (cells $\left.032-031 ; t_{(31)}=2.10 ; p<0.05\right)$. The G-group consisted of three subclusters. The neurons in the top (cells 003-138) and bottom (cells 012-135) subclusters showed significant QHCl side-band sensitivity not present in the 16 neurons in the middle subcluster (cells 017-098). The mean entropy score for the two G/Q-subclusters (0.86) was significantly higher than the average entropy score (0.74) for the G-subcluster that lacked QHCl sensitivity $\left(t_{(52)}=3.58 ; p<0.001\right)$.

The Q- and H-groups merged first in the dendrogram at a linkage distance of 0.83 based on their common sensitivity to $\mathrm{HCl}$ (see below). The N-group, which also contained several cells with significant side-band sensitivity to $\mathrm{HCl}$, merged next (1.10), and, finally, most distinct from the others, the G-group (1.30).

A different perspective on the relationship among gustatory neuron types is shown in the multidimensional plots of Figure 7, $A$ and $B$ (Clustan, version 7.1), which were calculated from the same correlation matrix of 142 cells described above. In both the two-dimensional (Fig. 7A) and three-dimensional (Fig. 7B) plots, the cells assumed an annulus because of their broad tuning. The dimensions are not defined, but in agreement with the dendrogram, the H-best and Q-best cells are more closely associated than any other of the four best-stimulus cell types. The loading of $\mathrm{NaCl}$-best, $\mathrm{HCl}$-best, and QHCl-best on the third dimension separated them to varying degrees from the glucose-best neurons, which formed an arc along the first and second dimensions of the space.

\section{Discussion}

Anatomical data imply that the mOFC should contain at least one area with a high concentration of gustatory neurons (Carmichael and Price, 1995b). This was indeed revealed in this electrophysiological investigation of BA $13 \mathrm{~m}$ and surrounding regions. Gustatory neurons were more concentrated in the mOFC than has been reported in either the AI (primary taste cortex) or the clOFC. Taste neurons in the mOFC have functional characteristics typical of both the insular cortex that projects to this region and of the clOFC, to which it projects (Carmichael and Price, 1996).

It has been reported in several studies that the posterior regions of the OFC contain taste-responsive neurons, but in each case, the percentage was small. Rolls et al. (1990) reported that only $49(1.6 \%)$ of 3120 cells within BA 120 responded to taste. Thorpe et al. (1983) have explored more medially, near the regions described in this study, and found 39 (7.9\%) taste cells within a sample of 494 neurons recorded from BAs $13 \mathrm{a}, 13 \mathrm{~m}$, and 13l. After testing some 1000 neurons throughout the OFC, Rolls et al. (1996) identified $65(6.5 \%)$ that were taste responsive. Rolls and Baylis (1994) tested 2000 cells within the AI and posterior OFC (BAs 11, 12, 13, and 14) and found 108 (5.4\%) that responded to taste. Although the precise number of cells recorded in each area was not reported, the majority were found within either the AI or further rostrally in BA 12o. The relatively low percentage of gustatory cells reported in these studies may be attributed to the fact that their surveys covered much larger areas of the OFC than the core and perimeter taste areas that are the focus of this study.

In this experiment, we identified a $12 \mathrm{~mm}^{2}$ region within the mOFC where $19.7 \%$ of the cells responded to sapid stimulation of the tongue. In the center of this core region, taste neurons accounted for as many as $28 \%$ of the cells tested. This high proportion does not result from our having used vastly different stimulus concentrations or a less stringent response criterion than those of previous investigators. The concentrations of glucose and $\mathrm{QHCl}$ in our basic stimulus array were the same as those used by SmithSwintosky et al. (1991) in the insula and those used by Rolls et al. (1990) in the clOFC. We tested $0.3 \mathrm{M} \mathrm{NaCl}$ versus the $1.0 \mathrm{M}$ used in the two studies cited above and $0.03 \mathrm{M} \mathrm{HCl}$ instead of $0.01 \mathrm{M}$.

The response criterion used in both the insula (SmithSwintosky et al., 1991) and clOFC (Rolls et al., 1990) required that the evoked response exceed the spontaneous rate by 2.33 SDs $(p<0.01)$. In this study, we felt it important to compensate for the possible influences of coincident tactile, thermal, and somatomotor stimulation or the nonspecific activity caused by our interactions with the monkey as we delivered each stimulus. Therefore, we compared the evoked response to that elicited by $\mathrm{dH}_{2} \mathrm{O}$ and demanded that the taste-elicited activity exceed this more elevated level by 1.96 SDs $(p<0.05)$. To verify that this had not resulted in a spuriously high taste cell count, the data were analyzed according to the former criterion (spontaneous, \pm 2.33 SDs). In the present study, the proportion of taste cells in the core was $19.7 \%$ when the $\mathrm{dH}_{2} \mathrm{O}$-based criterion was applied at $p<$ 0.05 and $19.9 \%$ when compared with the level of spontaneous activity at $p<0.01$. In the perimeter, the two percentages were 7.8 and 6.7, respectively. Thus, it does not appear that the high percentage of taste cells in the mOFC was caused by differences in either data collection or analysis among the studies.

\section{Taste processing in the human OFC}

Studies using positron emission tomography and functional MRI have identified numerous taste-related areas within the OFC 


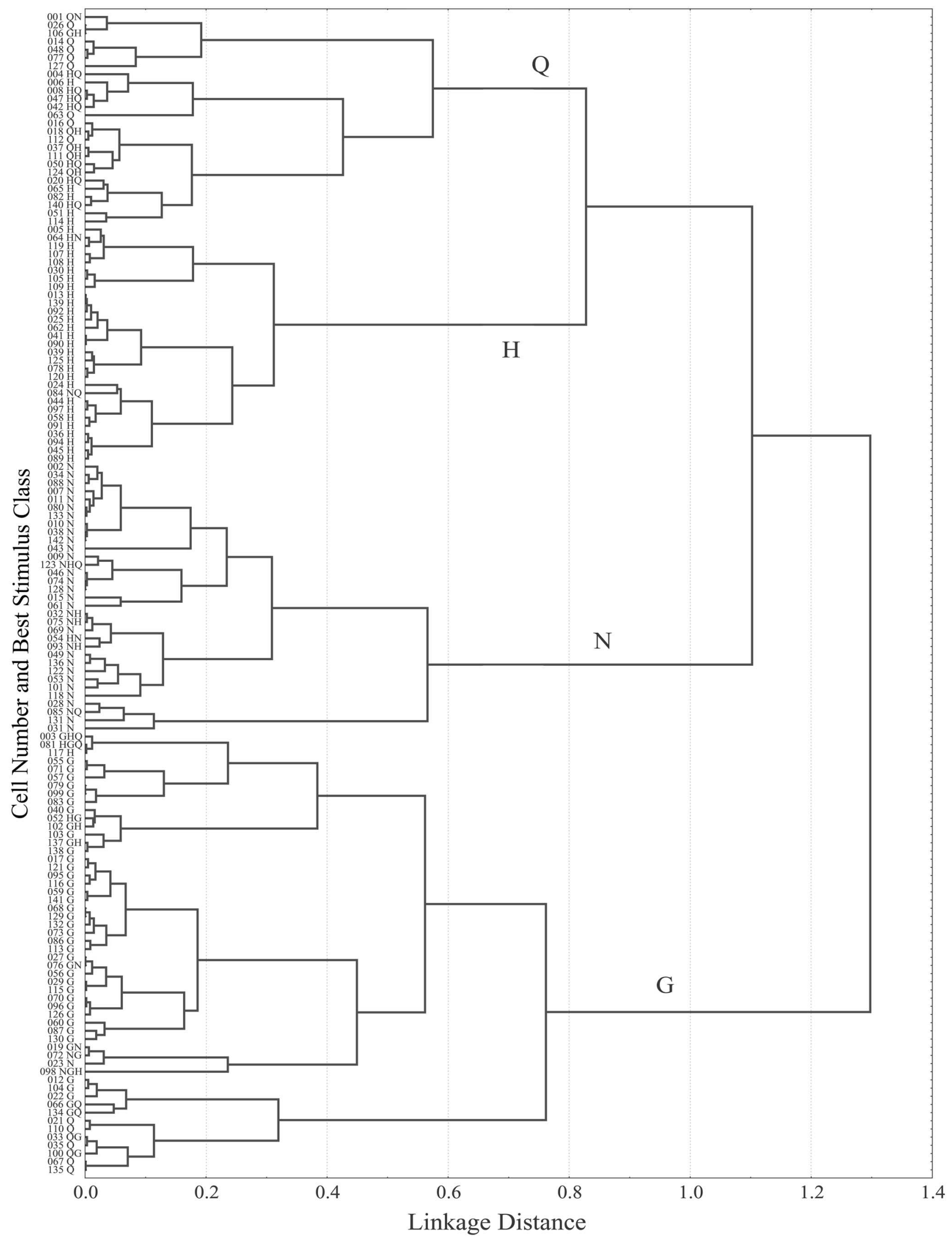

Figure 6. Response similarity based on cluster analysis of 142 neurons is shown as a dendrogram. The cell number indicates the order in which cells were recorded during the experiment. Each best-stimulus of the neuron, plus other stimuli that met the $80 \%$ criterion, is shown along the ordinate. $\mathrm{Q}, \mathrm{H}, \mathrm{N}$, and $\mathrm{G}$ indicate $\mathrm{Q}-, \mathrm{H}-\mathrm{N}, \mathrm{N}$, and $\mathrm{G}$-group, respectively. 
(Zald et al., 1998; Frank et al., 2003). Unlike the primary taste cortex, activation of these OFC areas is often dependent on either the affective valence of the stimulus (De Araujo et al., 2003; Small et al., 2003) or the subject's internal state, such as their level of hunger/satiety (Gautier et al., 2000; Del Parigi et al., 2002b) (but see LaBar et al., 2001; Del Parigi et al., 2002a). The existence of satiety-modulated taste neurons within the clOFC gives these findings in humans a secure neural footing (Rolls et al., 1989), but it is not known whether the distribution of hunger/ satiety-modulated neurons is as widespread as the human imaging data suggest. Tataranni et al. (1999), using positron emission tomography, have reported that most of the posterior OFC is activated by hunger and that part of the anterior OFC is driven by satiation. In the mOFC where the present data were recorded, only onehalf of the taste neurons are affected by satiety (our unpublished results). It is possible that the human imaging studies show a more widespread distribution for taste-related behavior, because their stimuli are activating areas related to reward or reward comparison, rather than taste per se (O’Doherty et al., 2002; Kringelbach and Rolls, 2004).

\section{Response properties of taste cells in the mOFC}

Spontaneous rate

The mean spontaneous rate of taste cells in the mOFC was $3.1 \pm 5.6$ spikes/s, nearly identical to the mean of 3.2 spikes/s reported for taste cells in the AI (Scott and Plata-Salaman, 1999) and nearly double the 1.6 spikes/s for those in the clOFC (Rolls et al., 1990).

\section{Multimodality}

Taste cells in the AI rarely respond to stimulation by other sensory modalities. Scott and Plata-Salamán (1999) described only the occasional unquantified neuron with both gustatory and olfactory sensitivity. In contrast, taste neurons in the clOFC are frequently responsive to olfactory, visual, and textural components of a stimulus. Rolls and Baylis (1994) found that 27\% (30 of 112 ) of taste cells also gave significant responses to olfactory (13.4\%) and visual (13.4\%) stimulation. Verhagen et al. (2003) reported that $31 \%$ (13 of 42 ) of taste cells in the clOFC also responded to the textural component of fat. In the mOFC, 45 of the 242 taste cells (18.6\%) responded to other modalities as well, most frequently to odors. Thus, although taste remains the dominant sensory modality in the mOFC, others impinge on these neurons, perhaps serving as the foundation for an integrated sense of flavor that is more fully expressed in the clOFC.

\section{Breadth of tuning}

There is a sharp dichotomy between the broadly tuned taste neurons encountered in the AI and the sharply defined responses of those in the clOFC. The mean BOT coefficient across some $800 \mathrm{AI}$ taste cells is 0.70 (Scott and Plata-Salamán, 1999), similar to those reported at the levels of the nucleus of the solitary tract $[0.87$ (Scott et al., 1986b)] and gustatory thalamus [0.73 (Pritchard et al., 1989)]. In contrast, taste cells in the clOFC have a mean coefficient of 0.39 (Rolls et al., 1990). Those in the mOFC respond similarly to those in the AI, with a mean BOT coefficient of 0.79 . This striking difference in entropy between the mOFC and the
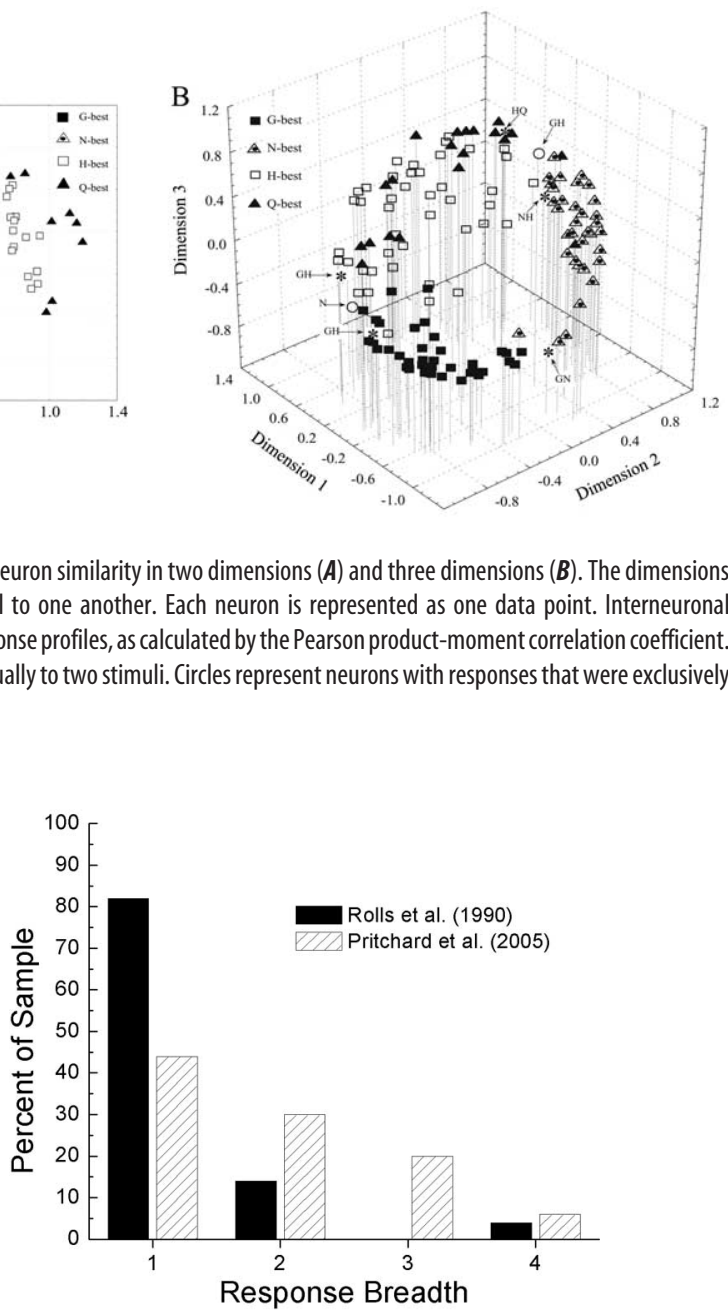

Figure 8. Percentage of taste neurons in the $\mathrm{mOFC}$ and the $\mathrm{ClOFC}$ that respond to one, two, three, or all four of the basic taste stimuli.

clOFC is evident when one compares the number of basic taste stimuli that drive neurons in each area. In the clOFC, $82 \%$ of the neurons respond to only one of the four basic taste stimuli; the corresponding percentage in the mOFC is 44\% (Fig. 8). Rolls et al. (1989) suggested that the unprecedented specificity in the clOFC is a necessary condition for the mechanism of sensoryspecific satiety to function. Sensory-specific satiety is the decrease in hedonic value for a particular food that develops as the subject becomes satiated to it (Rolls et al., 1983). Rolls and colleagues have fed monkeys to the point of satiety and found just such specific inhibition of taste neurons (Rolls et al., 1989), which corresponds to the loss of hedonic value for that taste, as measured behaviorally (Rolls et al., 1981). The narrow tuning of taste cells within the clOFC ensures that the hedonic value of other foods remains intact, which increases the probability that an animal, still hungry but sated on a particular food, will move to a different food/taste, thereby promoting a varied diet (Rolls et al., 1981). Thus, sensory-specific satiety may not require, but would be best served by, the narrowly tuned neurons found in the clOFC.

With a BOT of 0.79 , mOFC taste cells reflect the breadth of cells in the AI from which they receive input. (Note that the BOT coefficient would have been slightly higher in the $\mathrm{mOFC}$ if the criterion for taste had been based on dispersion around the spon- 
taneous activity level of each cell rather than the response to $\mathrm{dH}_{2} \mathrm{O}$.) Perhaps, for this reason, the phenomenon of sensoryspecific satiety in the mOFC, as demonstrated by reduced responsiveness to the taste of a stimulus on which the monkey is fed to satiety, is neither as robust nor as consistent as in the clOFC (our unpublished observations).

\section{Evoked activity levels}

In the $\mathrm{AI}$, neurons responding to glucose are most common at $60 \%$, but those responding to $\mathrm{QHCl}(56 \%), \mathrm{NaCl}(51 \%)$, and $\mathrm{HCl}(51 \%)$ are also well represented (Scott et al., 1986b). In the mOFC, the proportions change only modestly. The percentage of glucose-sensitive cells drops slightly to $50 \%$, whereas those responding to $\mathrm{NaCl}$ and $\mathrm{QHCl}$ decline to 43 and $40 \%$, respectively. The percentage of $\mathrm{HCl}$-sensitive neurons increased to $56 \%$. The difference between the clOFC and both the $\mathrm{AI}$ and $\mathrm{mOFC}$ is pronounced. In the clOFC, fully $82 \%$ of the taste cells respond to glucose; $\mathrm{NaCl}$-responsive (12\%), $\mathrm{HCl}$-responsive (12\%), and QHCl-responsive (8\%) neurons were poorly represented (Rolls et al., 1990). Thus, taste neurons within the mOFC, although still biased toward glucose, are nearly as evenly balanced among the basic stimuli as are those in the AI and much less oriented toward glucose than those in the clOFC.

\section{Insulo-orbital taste pathways}

The primary taste cortex is located in the crescent-shaped granular insula that straddles the rostral part of the circular sulcus (Pritchard et al., 1986). The ascending taste pathway from the insula to the OFC is likely to involve multiple synapses, because there are relatively few direct projections to the OFC from the rostrodorsal insula (Carmichael and Price, 1995b). The primary taste cortex projects to the dysgranular insula and agranular insula, two areas capable of serving as portals to the elaborate network of connections within the posterior OFC (Mufson and Mesulam, 1982; Mesulam and Mufson, 1985; Barbas, 1993; Carmichael and Price, 1995b, 1996). Guided by the anatomical description of the OFC by Carmichael and Price (1994, 1995b, 1996), we have explored the region of the mOFC in and immediately surrounding BA $13 \mathrm{~m}$ and have revealed a focus of robust gustatory activity. The taste cells encountered here display some of the features of primary cortical taste neurons in the AI (spontaneous levels of activity, broad tuning), others more in keeping with cells of the clOFC (multimodal responses), and some that are distinct from both (high density of taste cells, nearly equal responses to all four basic stimuli). We present this area as a discrete taste relay worthy of exploration for its involvement in gustatory neural coding, the amalgamation of sensory inputs to create flavor, and the hedonics that guide feeding behavior.

\section{References}

Bagshaw MH, Pribram KH (1953) Cortical organization in gustation (Macaca mulatta). J Neurophysiol 16:499-508.

Barbas H (1993) Organization of cortical afferent input to orbitofrontal area in the rhesus monkey. Neuroscience 56:841-864.

Baylis LL, Rolls ET, Baylis GC (1995) Afferent connections of the caudolateral orbitofrontal cortex taste area of the primate. Neuroscience 64:801-812.

Bieber SL, Smith DV (1986) Multivariate analysis of sensory data: a comparison of methods. Chem Senses 11:19-47.

Boudreau JC, Alev N (1973) Classification of chemoreceptive tongue units of the cat geniculate ganglion. Brain Res 54:157-175.

Carmichael ST, Price JL (1994) Architectonic subdivision of the orbital and medial prefrontal cortex in the macaque monkey. J Comp Neurol 346:366-402.
Carmichael ST, Price JL (1995a) Limbic connections of the orbital and medial prefrontal cortex in macaque monkeys. J Comp Neurol 363:615-641.

Carmichael ST, Price JL (1995b) Sensory and premotor connections of the orbital and medial prefrontal cortex of the macaque monkey. J Comp Neurol 363:642-664.

Carmichael ST, Price JL (1996) Connectional networks within the orbital and medial prefrontal cortex of macaque monkeys. J Comp Neurol 371:179-207.

Davis BJ, Jang T (1988) A Golgi analysis of the gustatory zone of the solitary tract in the adult hamster. J Comp Neurol 278:388-396.

De Araujo IET, Kringelbach ML, Rolls ET, McGlone F (2003) Human cortical responses to water in the mouth, and the effects of thirst. J Neurophysiol 90:1865-1876.

Del Parigi A, Gautier J-F, Chen K, Salbe AD, Ravussin E, Tataranni PA (2002a) Neuroimaging and obesity. Mapping the brain response to hunger and satiation in humans using positron emission tomography. Ann NY Acad Sci 967:389-397.

Del Parigi A, Chen K, Salbe AD, Gautier J-F, Ravussin E, Reiman EM, Tataranni PA (2002b) Tasting a liquid meal after prolonged fast is associated with preferential activation of the left hemisphere. NeuroReport 13:1141-1145.

Frank GK, Kaye WH, Carter CS Brooks S, May C, Fissell K, Stenger VA (2003) The evaluation of brain activity in response to taste stimuli-a pilot study and method for central taste activation as assessed by eventrelated fMRI. J Neurosci Methods 131:99-105.

Gautier J-F, Chen K, Salbe AD, Bandy D, Pratley RE, Heiman M, Ravussin E, Reiman EM, Tataranni PA (2000) Differential brain responses to satiation in obese and lean men. Diabetes 49:838-846.

Gibson AR, Hansma DI, Houk JC, Robinson FR (1984) A sensitive low artifact TMB procedure for the demonstration of WGA-HRP in the CNS. Brain Res 298:235-241.

Kringelbach ML, Rolls ET (2004) The functional neuroanatomy of the human orbitofrontal cortex: evidence from neuroimaging and neuropsychology. Prog Neurobiol 72:341-372.

LaBar KS, Gitenman DR, Parrish TB, Kim Y-H, Nobre AC, Mesulam M-M (2001) Hunger selectively modulates corticolimbic activation to food stimuli in humans. Behav Neurosci 115:493-500.

Mesulam M-M, Mufson EJ (1982) Insula of the Old World monkey. III: Efferent cortical output and comments on function. J Comp Neurol 212:38-52.

Mesulam M-M, Mufson EJ (1985) The insula of Reil in man and monkey. In: Cerebral cortex, Vol 4 (Peters A, Jones EG, eds), pp 179-226. New York: Plenum.

Miyaoka Y, Pritchard TC (1996) Responses of primate cortical neurons to unitary and binary taste stimuli. J Neurophysiol 75:396-411.

Mufson EJ, Mesulam M-M (1982) Insula of the Old World monkey. II: Afferent cortical input and comments on the claustrum. J Comp Neurol 212:23-37.

O’Doherty JP, Deichmann R, Critchley HD, Dolan RJ (2002) Neural responses during anticipation of a primary taste reward. Neuron 33:815-826.

Pritchard TC, Norgren R (2004) Gustatory system. In: The human nervous system (Paxinos G, Mai J, eds), pp 1171-1196. New York: Academic.

Pritchard TC, Hamilton RB, Morse JR, Norgren R (1986) Projections of thalamic gustatory and lingual areas in the monkey, Macaca fascicularis. J Comp Neurol 244:213-228.

Pritchard TC, Hamilton RB, Norgren R (1989) Neural coding of gustatory information in the thalamus of Macaca mulatta. J Neurophysiol 61:1-14.

Rolls BJ, Rolls ET, Rowe EA, Sweeney K (1981) Sensory specific satiety in man. Physiol Behav 27:137-142.

Rolls ET, Baylis LL (1994) Gustatory, olfactory, and visual convergence within the primate orbitofrontal cortex. J Neurosci 14:5437-5452.

Rolls ET, Rolls BJ, Rowe EA (1983) Sensory-specific and motivationspecific satiety for the sight and taste of food and water in man. Physiol Behav 30:185-192.

Rolls ET, Sienkiewicz ZJ, Yaxley S (1989) Hunger modulates the responses to gustatory stimuli of single neurons in the caudolateral orbitofrontal cortex of the macaque monkey. Eur J Neurosci 1:53-60.

Rolls ET, Yaxley S, Sienkiewicz ZJ (1990) Gustatory responses of single neurons in the caudolateral orbitofrontal cortex of the macaque monkey. J Neurophysiol 64:1055-1066.

Rolls ET, Critchley HD, Wakeman EA, Mason R (1996) Responses of neu- 
rons in the primate taste cortex to glutamate ion and to inosine $5^{\prime}$ monophosphate. Physiol Behav 59:991-1000.

Rolls ET, Critchley HD, Browning AS, Hernadi I, Lenard L (1999) Responses to the sensory properties of fat of neurons in the primate orbitofrontal cortex. J Neurosci 19:1532-1540.

Scott TR, Plata-Salamán CR (1999) Taste in the monkey cortex. Physiol Behav 67:489-511.

Scott TR, Yaxley S, Sienkiewicz ZJ, Rolls ET (1986a) Gustatory responses in the nucleus tractus solitarius of the alert cynomolgus monkey. J Neurophysiol 55:182-200.

Scott TR, Yaxley S, Sienkiewicz ZJ, Rolls ET (1986b) Gustatory responses in the frontal opercular cortex of the alert cynomolgus monkey. J Neurophysiol 56:876-890.

Scott TR, Plata-Salamán CR, Smith VL, Giza BK (1991) Gustatory neural coding in the monkey cortex: stimulus intensity. J Neurophysiol $65: 76-86$

Small DM, Gregory MD, Mak YE, Gitelman D, Mesulam M-M, Parrish T (2003) Dissociation of neural representation of intensity and affective valuation in human gustation. Neuron 39:701-711.

Smith DV, Travers JB (1979) A metric for the breadth of gustatory neurons. Chem Senses Flavour 4:215-229.

Smith-Swintosky VL, Plata-Salamán CR, Scott TR (1991) Gustatory neural coding in the monkey cortex: stimulus quality. J Neurophysiol 66:1156-1165.

Tataranni PA, Gautier J-F, Chen K, Uecker A, Bandy D, Salbe AD, Pratley RE, Lawson M, Reiman EM, Ravussin E (1999) Neuroanatomical correlates of hunger and satiation in humans using positron emission tomography. Proc Natl Acad Sci USA 96:4569-4574.

Thorpe SJ, Rolls ET, Maddison S (1983) The orbitofrontal cortex: neuronal activity in the behaving monkey. Exp Brain Res 49:93-115.

Travers JB (1988) Efferent projections from the anterior nucleus of the solitary tract of the hamster. Brain Res 457:1-11.

Verhagen JV, Rolls ET, Kadohisa M (2003) Neurons in primate orbitofrontal cortex respond to fat texture independently of viscosity. J Neurophysiol 90:1514-1525.

Whitehead MC, Frank ME (1983) Anatomy of the gustatory system in the hamster: central projections of the chorda tympani and the lingual nerves. J Comp Neurol 220:378-395.

Yaxley S, Rolls ET, Sienkiewicz ZJ, Scott TR (1990) Gustatory responses of single neurons in the insula of the macaque monkey. J Neurophysiol 63:689-700.

Zald DH, Lee JT, Fluegel KW, Pardo JV (1998) Aversive gustatory stimulation activates limbic circuits in humans. Brain 121:1143-1154. 